\title{
Radius Stabilization in a Supersymmetric Warped Compactification
}

\author{
Minoru Eto, ${ }^{1}$ Nobuhito Maru, ${ }^{2}$ and Norisuke Sakai ${ }^{1}$ \\ ${ }^{1}$ Department of Physics, Tokyo Institute of Technology, Tokyo 152-8551, JAPAN \\ ${ }^{2}$ Theoretical Physics Laboratory, RIKEN, Saitama 351-0198, JAPAN \\ meto@th.phys.titech.ac.jp, maru@postman.riken.go.jp, nsakai@th.phys.titech.ac.jp
}

(Dated: May 6, 2018)

\begin{abstract}
A supersymmetric (SUSY) model of radius stabilization is constructed for the $S^{1} / Z_{2}$ warped compactifications with a hypermultiplet in five dimensions. Requiring the continuity of scalar field across the boundaries, we obtain radius stabilization preserving SUSY, realizing the SUSY extension of the Goldberger-Wise mechanism. Even if we allow discontinuity of the $Z_{2}$ odd field across the boundary, we always obtain SUSY preservation but obtain the radius stabilization only when the discontinuity is fixed by other mechanisms.
\end{abstract}

PACS numbers: 11.25.-w, 11.30.Pb, 12.60.Jv

Keywords: Supersymmetry, Warped Extra Dimension, Radius Stabilization

\section{INTRODUCTION}

Motivated by branes in string theories, models with extra dimensions [1] have been proposed to offer another possible solution to the gauge hierarchy problem in recent years 2, 3], instead of the well-studied models with supersymmetry (SUSY) [4]. In these brane-world scenarios, the weak scale is derived from the four dimensional Planck scale through the large volume suppression [2] or through the warp factor [3] without fine-tuning of parameters. Many of these models have the compactification radius as one of the arbitrary parameters of the model, namely a moduli which are not determined by the dynamics of the model. For the scenarios to be relevant for nature, it is necessary to find the mechanism stabilizing the radius. For flat space models [2], a number of stabilization mechanisms have been proposed. An interesting possibility is to use the topological winding number as the origin of the stability [5]-[7]. Explicit models with topological stability have been worked out in four-dimensional models with four SUSY in flat space [5] - 7]. The model has also been successfully embedded into four-dimensional supergravity $[\underline{8}, 9]$ for warped compactifications [3]. Models with the topological stability in five-dimensions with eight SUSY are being worked out [10.

On the other hand, one of the popular models of radius stabilization for warped compactifications [3] is the model of Goldberger and Wise which uses a bulk scalar field 11]. They introduced appropriate potentials on the brane to pin-down the values of the scalar field. Then the bulk dynamics of the scalar field generates a potential to stabilize the radius. They have studied the limit where the backreaction to the warped geometry can be neglected. Similar stabilization mechanisms have been much studied using more general scalar fields 12, 13, 14].

SUSY is also quite useful in brane-world scenarios. The topological defects such as walls often break part of SUSY. Therefore the effective theories on the wall can possess half of SUSY of higher dimensional theories lead- ing to the well-studied $\mathcal{N}=1$ SUSY models in fourdimensions. SUSY also helps to obtain solutions of the topological defects needed for the brane-world scenarios, since the BPS equations for the partial SUSY conservation are much easier to solve. Even the warped compactification models using orbifold [3] have been realized as a zero-width limit of the domain wall solutions in supergravity 8, 9]. Considering SUSY warped compactification models is also well motivated from the viewpoint of SUSY flavor problem [15]. Separation of the hidden and the visible sectors in extra dimensions forbids the contact interactions between these two sectors causing flavor-violating scalar masses by the higher dimensional locality. Therefore, it is interesting to examine whether the Goldberger-Wise mechanism can be extended to SUSY theories. A simple SUSY model of radius stabilization is recently proposed and its related SUSY breaking phenomenology is also discussed [16].

The purpose of our paper is to propose a simple model of SUSY extention of the Goldberger-Wise mechanism of radius stabilization and to analyze the consequences. We find that SUSY is always preserved (four out of eight SUSY), with no additional contribution to the vacuum energy, justifying our assumption of neglecting backreaction to the background metric. We also find that the radius is stabilized as long as we insist on continuity across the orbifold fixed points for all the scalar fields including the $Z_{2}$ odd scalar field. If we allow a discontinuity of the $Z_{2}$ odd field at the boundary of $S^{1} / Z_{2}$ as a free parameter, we obtain solutions with single arbitrary parameter. Consequently the radius appears to become undetermined free parameters. We can understand the result by imagining a zero-width limit of a domain wall configuration [8, 17] which is made of the $Z_{2}$ odd scalar field. In the wall solution, the amount of the energy density generated by the wall is related to how rapidly the scalar field changes across the wall. In the zero-width limit, the discontinuities of the $Z_{2}$ odd field at both boundaries should be determined by the equations of motion for the scalar field if the appropriate dynamics is installed for the 
$Z_{2}$ odd scalar field to form the domain wall. Therefore we should consider the discontinuity to be a given parameter determined by (yet unspecified) wall dynamics. If we regard the discontinuity of the $Z_{2}$ odd field to be a given fixed input, the radius is uniquely determined.

In sec.2, we introduce our model and give solutions for generic situations with possible discontinuities for $Z_{2}$ odd scalar field. In sec.3, we give our results and discuss their physical implications.

\section{OUR MODEL}

We consider a SUSY theory with a hypermultiplet in five dimensions compactified on an orbifold $S^{1} / Z_{2}$. The metric is given by $[\underline{3}]$

$$
d s^{2}=e^{-2 r \sigma} \eta_{\mu \nu} d x^{\mu} d x^{\nu}+r^{2} d y^{2}, \sigma(y)=k|y|, 0 \leq y \leq \pi,
$$

where $\mu, \nu=0, \cdots, 3$, and $r$ is the $S^{1}$ compactification radius of the extra dimension $x^{4} \equiv y$. Since we assume the backreaction from the bulk scalar field is negligible [1], the background warped (AdS) geometry (10) is fixed and the supergravity multiplet is treated as frozen and nondynamical. Using the four SUSY superfield formalism [18, 19, 20, 21, 22], the Lagrangian is given by [23]

$$
\begin{aligned}
\mathcal{L}_{5}= & \int d^{4} \theta r e^{-2 r \sigma}\left[|H|^{2}+\left|H^{c}\right|^{2}\right] \\
& +\left[\int d ^ { 2 } \theta e ^ { - 3 r \sigma } \left\{H^{c}\left(\partial_{y}-\left(\frac{3}{2}-c\right) r \sigma^{\prime}\right) H\right.\right. \\
& \left.\left.+\delta(y) W_{0}+\delta(y-\pi) W_{\pi}\right\}+ \text { h.c. }\right]
\end{aligned}
$$

where the prime denotes the differentiation with respect to $y$, and the constant $c$ specifies the bulk mass of hypermultiplets. The chiral scalar superfields $H$ and $H^{c}$ in the four SUSY notation form a hypermultiplet of eight SUSY. Since eight SUSY does not allow superpotentials among hypermultiplets in the bulk, we can introduce the superpotentials only on the boundaries $y=0, \pi$ (orbifold fixed points) which are denoted as $W_{0}, W_{\pi}$, respectively. The orbifolding on $S^{1} / Z_{2}$ breaks the eight SUSY maintaining only four SUSY. We shall assign even $Z_{2}$ parity to $H$, odd $Z_{2}$ parity to $H^{c}$, respectively. Since only the even field can have nonvanishing values on the boundaries, the boundary superpotential can have only parity even field $H$.

For simplicity, we shall take the quadratic boundary superpotential with a unique SUSY vacuum

$$
W_{0, \pi}=\frac{1}{2} H^{2}-v_{0, \pi} H
$$

where $v_{0, \pi}$ are constants with mass dimension $3 / 2$. It is useful to make a rescaling

$$
\left(H, H^{c}\right) \rightarrow e^{r \sigma}\left(H, H^{c}\right) .
$$

Then the Lagrangian becomes

$$
\begin{aligned}
\mathcal{L}_{5} \rightarrow & \int d^{4} \theta r\left(|H|^{2}+\left|H^{c}\right|^{2}\right) \\
& +\left[\int d ^ { 2 } \theta \left\{e^{-r \sigma} H^{c} \partial_{y} H+e^{-r \sigma}\left(c-\frac{1}{2}\right) r \sigma^{\prime} H^{c} H\right.\right. \\
& +\delta(y)\left(\frac{1}{2} e^{-r \sigma} H^{2}-v_{0} e^{-2 r \sigma} H\right) \\
& \left.\left.+\delta(y-\pi)\left(\frac{1}{2} e^{-r \sigma} H^{2}-v_{\pi} e^{-2 r \sigma} H\right)\right\}+ \text { h.c. }\right] .(5)
\end{aligned}
$$

It is straightforward to derive the auxiliary fields part of Lagrangian,

$$
\begin{aligned}
\mathcal{L}_{\text {aux }}= & r\left(|F|^{2}+\left|F^{c}\right|^{2}\right) \\
& +\left[e ^ { - r \sigma } \left\{F^{c} \partial_{y} H+r \sigma^{\prime} H^{c} F-\partial_{y} H^{c} F\right.\right. \\
& \left.+\left(c-\frac{1}{2}\right) r \sigma^{\prime}\left(F^{c} H+H^{c} F\right)\right\} \\
& +\delta(y)\left(e^{-r \sigma} H-v_{0} e^{-2 r \sigma}\right) F \\
& \left.+\delta(y-\pi)\left(e^{-r \sigma} H-v_{\pi} e^{-2 r \sigma}\right) F+\text { h.c. }\right] \\
= & -r\left(|F|^{2}+\left|F^{c}\right|^{2}\right) .
\end{aligned}
$$

In the second equality, we used the equations of motion (EOM) for auxiliary fields derived from Eq. (6)

$$
\begin{aligned}
F^{*} & =\frac{e^{-r \sigma}}{r}\left[\partial_{y} H^{c}-\left(c+\frac{1}{2}\right) r \sigma^{\prime} H^{c}-e^{r \sigma} W_{b}\right] \\
& =\frac{e^{-r \sigma}}{r}\left[e^{\left(c+\frac{1}{2}\right) r \sigma} \partial_{y}\left(e^{-\left(c+\frac{1}{2}\right) r \sigma} H^{c}\right)-e^{r \sigma} W_{b}\right] \\
F^{c *} & =-\frac{e^{-r \sigma}}{r}\left[\partial_{y} H+\left(c-\frac{1}{2}\right) r \sigma^{\prime} H\right] \\
& =-\frac{e^{-r \sigma}}{r}\left[e^{-\left(c-\frac{1}{2}\right) r \sigma} \partial_{y}\left(e^{\left(c-\frac{1}{2}\right) r \sigma} H\right)\right]
\end{aligned}
$$

where

$$
\begin{aligned}
W_{b}= & \delta(y)\left(e^{-r \sigma} H-v_{0} e^{-2 r \sigma}\right) \\
& +\delta(y-\pi)\left(e^{-r \sigma} H-v_{\pi} e^{-2 r \sigma}\right) \\
\equiv & \delta(y) \tilde{W}_{0}+\delta(y-\pi) \tilde{W}_{\pi} .
\end{aligned}
$$

Here and the following, we shall use the same notation for scalar fields as the superfields. It is important to notice that the auxiliary field $F$ in Eq. (8) contains delta function in general which introduces singular interaction terms like $(\delta(y))^{2}$ as noted previously [20].

In conformity with the warped metric compactifications in Eq.(11), we are interested in the configurations of the scalar fields $H, H^{c}$ as functions of extra dimensional coordinate $y$ only. The scalar field $H$ with the even $Z_{2}$ parity does not vanish at the boundaries. However, the scalar field $H^{c}(y)$ with odd $Z_{2}$ parity has to vanish at the boundaries. To make this point clear, we rewrite the parity odd field $H^{c}(y)$ in terms of a parity even field $h^{c}(y)$ as

$$
H^{c}(y)=\hat{\varepsilon}(y) h^{c}(y)
$$


where $\hat{\varepsilon}(y)$ is a sign function of $y$

$$
\hat{\varepsilon}(y) \equiv \begin{cases}-1, & \text { for }-\pi<y<0 \\ 0, & \text { for } y=0, \pm \pi \\ 1, & \text { for } 0<y<\pi\end{cases}
$$

From physical grounds, we should consider field configurations of the physical scalar fields $H(y), H^{c}(y)$ which are continuous across the boundaries. This implies that we need to require

$$
h^{c}(0)=h^{c}(\pi)=0 .
$$

In special circumstances like the zero-width limit of domain wall configurations, the odd $Z_{2}$ parity field $H^{c}$ can have a discontinuity across the boundaries. In order to examine such a general situation later, we shall temporarily allow discontinuities across the boundaries for $H^{c}$, corresponding to a finite nonvanishing values of $h^{c}(0)$ and $h^{c}(\pi)$. Apart from this subtlety, $h^{c}(y)$ is assumed to be a continuous 24] and parity even function of $y$. Then, Eq. (8) can be rewritten as

$$
F^{*}=\frac{e^{-r \sigma}}{r}\left[e^{\left(c+\frac{1}{2}\right) r \sigma} \partial_{y}\left(e^{-\left(c+\frac{1}{2}\right) r \sigma} \hat{\varepsilon}(y) h^{c}(y)\right)-e^{r \sigma} W_{b}\right] .
$$

As far as $H, H^{c}$ depend on $y$ only, it is sufficient to consider only the auxiliary fields part of the Lagrangian (17). Then the EOM for $H^{c}$ is given by

$$
\begin{aligned}
0 & =\frac{\partial \mathcal{L}}{\partial H^{c *}}-\partial_{y} \frac{\partial \mathcal{L}}{\partial \partial_{y} H^{c *}}=\frac{1}{r} e^{-\left(c+\frac{1}{2}\right) r \sigma} \partial_{y}\left[e^{\left(c-\frac{3}{2}\right) r \sigma}\right. \\
& \left.\times\left\{e^{\left(c+\frac{1}{2}\right) r \sigma} \partial_{y}\left(e^{-\left(c+\frac{1}{2}\right) r \sigma} \hat{\varepsilon}(y) h^{c}(y)\right)-e^{r \sigma} W_{b}\right\}\right](1
\end{aligned}
$$

The singular part of the equations of motion (16) contains $\partial_{y} \delta(y), \partial_{y} \delta(y-\pi)$ originating from $\frac{\partial W_{b}}{\partial y}$ and $\partial_{y}^{2} \hat{\varepsilon}(y)$ and reads

$$
\begin{aligned}
0= & \frac{e^{-2 r \sigma}}{r}\left[\partial_{y} \delta(y)\left(2 h^{c}(0)-\tilde{W}_{0}\right)\right. \\
& \left.+\partial_{y} \delta(y-\pi)\left(-2 h^{c}(\pi)-e^{r k \pi} \tilde{W}_{\pi}\right)\right] .
\end{aligned}
$$

Thus we obtain the following boundary conditions

$$
\begin{aligned}
2 h^{c}(0) & =\tilde{W}_{0}=H(0)-v_{0}, \\
-2 h^{c}(\pi) & =e^{r k \pi} \tilde{W}_{\pi}=H(\pi)-e^{-r k \pi} v_{\pi} .
\end{aligned}
$$

Taking these boundary conditions into account, we can immediately see that the auxiliary field $F$ contains no delta functions,

$$
F^{*}=\hat{\varepsilon}(y) \frac{e^{\left(c-\frac{1}{2}\right) r \sigma}}{r} \partial_{y}\left(e^{-\left(c+\frac{1}{2}\right) r \sigma} h^{c}\right) .
$$

It is interesting to observe that the pining of the scalar field values $H(0), H(\pi)$ at boundaries arises in order to satisfy the EOM at the boundary, resulting in no singular terms in the auxiliary field as a result. This is in contrast to the Goldberger-Wise model without SUSY which requires a sharp potential well by tuning coupling parameters. Returning to the remaining EOM of $h^{c}$

$$
0=\frac{1}{r} e^{-\left(c+\frac{1}{2}\right) r \sigma} \partial_{y}\left\{\hat{\varepsilon}(y) e^{(2 c-1) r \sigma} \partial_{y}\left(e^{-\left(c+\frac{1}{2}\right) r \sigma} h^{c}\right)\right\},
$$

we find the general solution

$h^{c}(y)= \begin{cases}-\frac{C_{1}}{(2 c-1) r k} e^{-\left(c-\frac{3}{2}\right) r \sigma}+C_{2} e^{\left(c+\frac{1}{2}\right) r \sigma}, & \text { for } c \neq \frac{1}{2}, \\ \left(C_{1}|y|+C_{2}\right) e^{r \sigma}, & \text { for } c=\frac{1}{2},\end{cases}$

where $C_{1,2}$ are integration constants. The auxiliary field $F(y)$ is given by

$$
F^{*}(y)=C_{1}(\hat{\varepsilon}(y))^{2} \frac{e^{-\left(c-\frac{1}{2}\right) r \sigma}}{r}
$$

irrespective of $c$. Note that $F$ can be non-zero only in the bulk and trivially vanishes at the boundaries, because of $\hat{\varepsilon}(y=0, \pi)=0$, except when it is multiplied by singular functions like delta functions.

Let us turn to the EOM for $H$,

$$
\begin{aligned}
0= & \frac{1}{r}\left[e^{\left(c-\frac{1}{2}\right) r \sigma} \partial_{y}\left\{e^{-(2 c+1) r \sigma} \partial_{y}\left(e^{\left(c-\frac{1}{2}\right) r \sigma} H\right)\right\}\right. \\
& \left.+r F^{*}\left\{\delta(y)+e^{-r \sigma} \delta(y-\pi)\right\}\right] .
\end{aligned}
$$

Since $F^{*}$ in Eq. (24) is multiplied by a delta function, it gives nonvanishing contributions at the fixed points $y=0, \pi$ in spite of the square of the sign function in Eq. (23) [25], 26] :

$$
\begin{aligned}
(\varepsilon(y))^{2 n} \delta(y) & =\frac{1}{2 n+1} \delta(y), \\
(\varepsilon(y))^{2 n+1} \delta(y) & =0, \quad n=0,1,2, \cdots .
\end{aligned}
$$

Then, the remaining EOM for $H$ reads

$$
\begin{aligned}
0 & =e^{\left(c-\frac{1}{2}\right) r \sigma} \partial_{y}\left\{e^{-(2 c+1) r \sigma} \partial_{y}\left(e^{\left(c-\frac{1}{2}\right) r \sigma} H\right)\right\} \\
& +\frac{C_{1}}{3}\left\{\delta(y)+e^{\left(c+\frac{1}{2}\right) r k \pi} \delta(y-\pi)\right\}
\end{aligned}
$$

The EOM in the bulk $(y \neq 0, \pi)$ becomes

$$
e^{-(2 c+1) r \sigma} \partial_{y}\left(e^{\left(c-\frac{1}{2}\right) r \sigma} H\right)=C_{3} \hat{\varepsilon}(y),
$$

with an integration constant $C_{3}$. The $Z_{2}$ parity transformation property requires the sign function $\hat{\varepsilon}(y)$ in the right hand. However, we still need to examine the equations of motion (26) at the boundaries. The solution in the bulk (27) gives delta functions at the boundaries

$$
\partial_{y}\left(e^{-(2 c+1) r \sigma} \partial_{y}\left(e^{\left(c-\frac{1}{2}\right) r \sigma} H\right)\right)=2 C_{3}(\delta(y)-\delta(y-\pi)) .
$$

The equations of motion (26) is satisfied at the boundaries only when these delta functions are cancelled each other : the delta function at $y=0$ cancells if

$$
0=2 C_{3}+\frac{C_{1}}{3}
$$


the delta function at $y=\pi$ cancells if

$$
0=-2 C_{3} e^{-\left(c-\frac{1}{2}\right) k \pi}+\frac{C_{1}}{3} e^{\left(c+\frac{1}{2}\right) r k \pi} .
$$

These two conditions together imply

$$
C_{1}=C_{3}=0 .
$$

The solution of Eq.(27) with $C_{3}=0$ is given by

$$
H(y)=C_{4} e^{-\left(c-\frac{1}{2}\right) r \sigma} .
$$

The solution of the Eqs. (9) and (27) with $C_{3}=0$ gives

$$
F^{c *}(y)=0 .
$$

The result $C_{1}=0$ also implies the vanishing auxiliary field $F$ in Eq. 223.

$$
F(y)=0,
$$

and the $Z_{2}$ odd scalar field in Eq.(22) as

$$
H^{c}(y)=\hat{\varepsilon}(y) h^{c}(y)=\hat{\varepsilon}(y) C_{2} e^{\left(c+\frac{1}{2}\right) r \sigma} .
$$

Therefore we find that both auxiliary fields $F$ and $F^{c}$ vanish, and that SUSY is always preserved.

Using Eqs. (32) and (35), we can determine the remaining two integration constants $C_{2}, C_{4}$ from the boundary conditions (18) and (19) as

$$
\begin{aligned}
C_{2} & =\frac{-v_{0} e^{-2 c r k \pi}+v_{\pi} e^{-\left(c+\frac{3}{2}\right) r k \pi}}{2\left(1+e^{-2 c r k \pi}\right)}, \\
C_{4} & =\frac{v_{0} e^{c r k \pi}+v_{\pi} e^{-\frac{3}{2} r k \pi}}{1+e^{-2 c r k \pi}}
\end{aligned}
$$

\section{RESULTS}

Since the auxiliary fields $F, F^{c}$ vanish, SUSY is always preserved. As we stated in the previous section, we primarily consider that the physical scalar fields $H$, $H^{c}$ should be continuous across the boundaries $y=0, \pi$. This implies that the field $h^{c}(y)$ should vanish at the boundaries : $h^{c}(0)=h^{c}(\pi)=0$. Then the boundary conditions (18) and (19) force the boundary values $H(0)$ and $H(\pi)$ of $Z_{2}$ even field $H(y)$ to settle at the minimum of the boundary superpotential

$$
H(0)=v_{0}, \quad H(\pi)=v_{\pi} e^{-r k \pi},
$$

as in the non-SUSY case 11]. Combining Eqs. (32) and (38), we obtain

$$
v_{0}=v_{\pi} e^{\left(c-\frac{3}{2}\right) r k \pi}
$$

which determines the radius. The radius stabilization is thus achieved in our SUSY model. Moreover, the vanishing contribution to the vacuum energy justifies our assumption of no backreaction to Einstein equation for the metric. These result precisely realizes the objective of the Goldberger-Wise model. In our SUSY model, however, the field values $H(0), H(\pi)$ are fixed at the boundaries by their EOM without tuning parameters of the potential in contrast to the non-SUSY Goldberger-Wise model. We also find that $C_{2}=0$ from Eq.(35) with $h^{c}(0)=h^{c}(\pi)=0$, and that

$$
h^{c}(y)=0 .
$$

On the other hand, if we allow nonvanishing discontinuities of the $Z_{2}$ odd field $H^{c}(y)$ across the boundaries, we obtain more complications. Even in this case, the equations of motion requires these discontinuities $h^{c}(0), h^{c}(\pi)$ to be related as given in Eqs.(35) and (36) :

$$
h^{c}(\pi)=h^{c}(0) e^{\left(c+\frac{1}{2}\right) r k \pi} .
$$

Then the solution in the previous section contains single arbitrary parameter, say $h^{c}(0)$ undetermined. Therefore the radius is determined only by fixing the discontinuity $h^{c}(0)$. In order to understand the physical significance of these discontinuities, it should be useful to consider the zero-width limit of domain wall solutions with a scalar field [8, 17]. Since a domain wall consists of a kink of scalar field in the extra dimension, the scalar field usually changes sign at the boundaries. This is precisely a feature of the $Z_{2}$ odd scalar field. Therefore it is tempting to identify the $Z_{2}$ odd scalar field with the scalar field forming the wall. In the zero-width limit, the wall energy is concentrated at the boundary as a delta-function and should be related to the discontinuity of the scalar field across the boundary. Therefore we believe that the amount of the discontinuity across the boundary $h^{c}(0)$ (and $h^{c}(\pi)$ ) should be determined by the yet unspecified dynamics to form the domain wall. Provided such a microscopic description is given, this discontinuity is a fixed input parameter in our situation. Then the radius of compactification is determined using the fixed parameter as the input.

It may be instructive to compare our model with a model admitting an exact two wall solution stabilized by a winding number [5, 6], which was embedded into supergravity in four dimensions 8]. In this model with winding number, a chiral scalar field serves as a $Z_{2}$ odd field to form domain walls with the symmetry $S^{1} / Z_{2}$. If the width of the wall is finite, the two wall configuration is found to be non-BPS (SUSY is completely broken) and the radius is stabilized [9]. In the limit of vanishing width (keeping wall tension fixed), however, the $Z_{2}$ odd scalar field of this model has no discontinuities across the orbifold boundaries leaving only boundary vacuum energy as a remnant. Then the model reduces to the Randall-Sundrum model [3], and the scalar field is frozen in the zero-width limit without any other field available for the Goldberger-Wise type mechanism of radius stabilization to work. In fact, the two-wall solution can be 
regarded as a BPS configuration preserving half of the bulk SUSY 25, 27], and the radius is undetermined in the zero width limit [8]. The scalar field forming the wall acts as a stabilizer field only for finite width of the wall, with fully broken SUSY. On the contrary, our present SUSY model of radius stabilization has the $Z_{2}$ even field $H$ and the boundary superpotential, which provide the stabilization mechanism preserving SUSY (assuming continuity of fields).

The advantages of our model are as follows. First, the stabilization of radius is maintained perturbatively, since the stabilization condition is determined by the F-flatness conditions. Even if the corrections to Kähler potential are considered, the conditions remain unchanged as long as the Kähler metric is non-singular and positive definite after quantum corrections. Second, we do not necessarily need to tune the warp factor to be $e^{-r k \pi} \sim 10^{-16}$ since the hierarchy problem can be solved by SUSY preserved on the boundaries. This fact offers more possibilities for the viable model construction. Third, as we mentioned in Introduction, the radius stabilization in SUSY models are required to address the SUSY flavor problem in the context of the brane world. Supersymmetric radius stabilization is phenomenologically favored as discussed in [16.

Finally, we comment on the difference between our model and the model in [16]. In the model of [16], there are always the discontinuities of the $Z_{2}$ odd scalar field across the boundaries because of the boundary superpotential linear in $Z_{2}$ even chiral superfield $H$. But in our model, the case without discontinuities is also allowed as mentioned in the text.
In summary, we have proposed a simple model of stabilizing the compactification radius in SUSY warped compactifications with a hypermultiplet. By solving the equations of motion, we find that SUSY is always preserved. If the $Z_{2}$ odd scalar field of the hypermultiplet has no discontinuities across the boundaries, the $Z_{2}$ even scalar field settles at the minimum of the boundary superpotential, and the radius is determined by Eq. (39). This corresponds to a SUSY version of the GoldbergerWise model. More generally, if we allow discontinuities of the $Z_{2}$ odd scalar field across the boundaries, the $Z_{2}$ even scalar field does not necessarily settle at the minimum of the boundary superpotential, and the radius is stabilized only after fixing the discontinuity at the boundary by using the yet unspecified dynamics of forming the domain wall.

\section{Acknowledgements}

One of the authors (M.E.) gratefully acknowledges support from the Iwanami Fujukai Foundation and from a 21st Century COE Program at Tokyo Tech "NanometerScale Quantum Physics" by the Ministry of Education, Culture, Sports, Science and Technology. This work is supported in part by Grant-in-Aid for Scientific Research from the Ministry of Education, Culture, Sports, Science and Technology, Japan No.13640269 and No.16028203 for the priority area "Origin of mass" (NS) and by Special Postdoctoral Researchers Program at RIKEN (NM).
[1] P. Horava and E. Witten, Nucl. Phys. B460, 506 (1996) hep-th/9510209.

[2] N. Arkani-Hamed, S. Dimopoulos and G. R. Dvali, Phys. Lett. B429, 263 (1998) hep-ph/9803315; I. Antoniadis, N. Arkani-Hamed, S. Dimopoulos and G. R. Dvali, Phys. Lett. B436, 257 (1998) hep-ph/9804398.

[3] L. Randall and R. Sundrum, Phys. Rev. Lett. 83, 3370 (1999) hep-ph/9905221; Phys. Rev. Lett. 83, 4690 (1999) hep-th/9906064.

[4] S. Dimopoulos and H. Georgi, Nucl. Phys. B193, 150 (1981); N. Sakai, Z. f. Phys. C11, 153 (1981); E. Witten, Nucl. Phys. B188, 513 (1981); S. Dimopoulos, S. Raby, and F. Wilczek, Phys. Rev. D24, 1681 (1981).

[5] N. Maru, N. Sakai, Y. Sakamura, and R. Sugisaka, Phys. Lett. B496, 98 (2000) hep-th/0009023.

[6] N. Maru, N. Sakai, Y. Sakamura, and R. Sugisaka, Nucl. Phys. B616, 47 (2001) hep-th/0107204; N. Maru, N. Sakai, Y. Sakamura, and R. Sugisaka, the Proceedings of the 10th Tohwa international symposium on string theory, American Institute of Physics, 607, pages 209-215, (2002) hep-th/0109087; N. Maru, N. Sakai, Y. Sakamura, and R. Sugisaka, "SUSY Breaking by stable nonBPS configurations", to appear in the Proceedings of the Corfu Summer Institute on Elementary particle Physics, Corfu, September 2001 hep-th/0112244.
[7] N. Sakai and R. Sugisaka, Int. J. Mod. Phys. A17, 4697 (2002) 4697 hep-th/0204214.

[8] M. Eto, N. Maru, N. Sakai, and T. Sakata, Phys. Lett. B553, 87 (2003) hep-th/0208127.

[9] M. Eto, N. Maru, and N. Sakai, Nucl. Phys. B673, 98 (2003) hep-th/0307206.

[10] M. Eto, N. Maru, and N. Sakai, "Non-BPS Walls and Their Stability in 5D Supersymmetric Theory", hep-th/0404114.

[11] W.D. Goldberger and M.B. Wise, Phys. Rev. Lett. 83, 4922 (1999) hep-th/9907447.

[12] O. DeWolfe, D.Z. Freedman, S.S. Gubser, and A. Karch, Phys. Rev. D62, 046008 (2000) hep-th/9909134.

[13] K. Skenderis, and P.K. Townsend, Phys. Lett. B468, 46 (1999) hep-th/9909070.

[14] A. Lewandowski, M.J. May, and R. Sundrum, Phys. Rev. D67, 024036 (2003) hep-th/0209050; I. Brevik, K. Ghoroku, M. Yahiro, "Radius stabilization and brane running in RS1 model", hep-th/0402176; A. A. Saharian, M. R. Setare, Phys. Lett. B552, 119 (2003) hep-th/0207138.

[15] L. Randall and R. Sundrum, Nucl. Phys. B557, 79 (1999) hep-th/9810155.

[16] N. Maru and N. Okada, hep-th/0312148

[17] M. Arai, S. Fujita, M. Naganuma, and N. Sakai, Phys. 
Lett. B556, 192 (2003) hep-th/0212175.

[18] N. Arkani-Hamed, T. Gregoire and J. Wacker, JHEP 0203, 055 (2002) arXiv:hep-th/0101233.

[19] N. Marcus, A. Sagnotti and W. Siegel, Nucl. Phys. B224, 159 (1983).

[20] E. A. Mirabelli and M. E. Peskin, Phys. Rev. D58, 065002 (1998) hep-th/9712214.

[21] A. Hebecker, Nucl. Phys. B632, 101 (2001) hep-th/0112230.

[22] K. Kakimoto, and N. Sakai, Phys. Rev. D68, 065005 (2003) hep-th/0306077.
[23] D. Marti and A. Pomarol, Phys. Rev. D64, 105025 (2001) hep-th/0106256.

[24] First derivative may be discontinuous, as seen in the solution in Eq. (22).

[25] R. Altendorfer, J. Bagger and D. Nemeschansky, Phys. Rev. D63 (2001) 125025, hep-th/0003117.

[26] K.A. Meissner, H.P. Nilles and M. Olechowski, Acta Phys. Polon. B33 (2002) 2435, hep-th/0205166.

[27] A. Falkowski, Z. Lalak and S. Pokorski, Phys. Lett. B491 (2000) 172, hep-th/0004093. 